\title{
Effect of Uniform and Non-Uniform Hot Wall Temperature on Species Separation in a Tilting Cell
}

\author{
Mouna Touiker ${ }^{1}$, and Abdeslam Omara ${ }^{2, *}$ \\ ${ }^{1}$ University of 20 août 55 , Skikda, Algeria \\ ${ }^{2}$ University of Mentouri Brothers Constantine 1, Constantine, Algeria
}

\begin{abstract}
This work deals with the Soret separation of a binary liquid mixture (Water $60 \mathrm{wt} \%$-Ethanol 40 wt \%). The studied configuration is a tilted rectangular cavity of large aspect ratio $A$. It is assumed that the right wall is heated uniformly or non-uniformly, while the left wall is maintained at a constant cold temperature. The top and bottom walls are well insulated. A developed program (based on the finite-volume method and the semi-implicit method for pressure-linked equations algorithm) was utilized to numerically solve the governing Navier-Stokes equations, the energy and the species equations with the associated boundary conditions. The relevant parameters in this study are the tilting angle $(\alpha)$, the aspect ratio $(A)$, the mass Rayleigh number $\left(R a_{m}\right)$ and the Lewis number $(L e)$.
\end{abstract}

\section{Introduction}

The Soret effect refers to the transport of mass induced in a fluid mixture by a temperature gradient. This type of flow has applications in many branches of sciences and engineering like isotope separation. Species separation of binary mixture in the presence of mixed convection was analyzed analytically and numerically by Khouzam et al. [1]. They found that the mass concentration gradient is only function of two new dimensionless parameters, namely mass Rayleigh number and mass Péclet number. Bennacer et al. [2] have analyzed the influence of the Soret effect on the convection in a horizontal porous layer under crossed temperature and concentration gradients. Numerical and analytical results for the case of a horizontal rectangular cavity filled with a binary mixture under the influence of a horizontal thermal gradient were presented by Elhajjar et al. [3]. They have obtained appreciable separations for reasonable values of the thickness of the cavity of the order of $2 \mathrm{~mm}$ and for lengths of the order of $500 \mathrm{~mm}$, which represents an advantage compared to the vertical cavity which requires very small thicknesses for the same order of magnitude of separations. Elhajjar et al. [4] have presented a theoretical and numerical study of species separation in an inclined porous cavity. Platten et al. [5] have demonstrated that the inclination of a thermo-gravitational column increases the molecular separation. Ouadhani et al. [6] have studied the effect of mechanical vibrations on the separation of a binary mixture saturating a porous horizontal cavity. In this work the low horizontal plate is submitted to constant uniform heat flux and the top one is maintained to a constant temperature. They have observed that the vertical vibrations have a stabilizing effect on convection, and that the vibrations could be used to decrease the value of the separation factor leading to the unicellular flow, allowing separation in the binary mixture in a horizontal cell for a broad range of binary mixtures. Yang and Ripoll [7] propose a new theoretical expression for the Soret coefficient $S_{T}$, which applies equally well to molecular mixtures and colloidal suspensions. The new expression is validated by means of computer simulations and results to be especially suitable for studying the thermal diffusion of dilute solutions. Shevtsova et al. [8] present a threedimensional 3D time-dependent numerical study describing the transient stage of Soret driven instability in the system heated from above. Henry and Roux [9] studied the Soret separation in a differentially heated elongated cylinder $(A=3)$ slightly inclined (tilted at a small angle $\alpha)$ with moderate Prandtl number $(\mathrm{Pr}=0.6)$, high Schmidt number $(\mathrm{Sc}=60)$ and positive separation $S$. They found that the smaller the Soret coefficient is, the smaller the inclination defect $\boldsymbol{\alpha}$ has to be to allow a good separation rate.

Raju et al. [10] numerically studied the Soret effects on separation of a binary mixture due to natural convection between heated inclined plates under the influence of transverse magnetic field. They have found that the separation of the binary fluid mixture increases with the increase of the tilting angle that the plates make with the horizontal. Species separation in a binary fluid saturating a horizontal porous layer subjected to cross heat fluxes is investigated numerically and analytically by Yacine et al. [11]. The results were obtained for a wide range of governing parameters, namely the thermal Rayleigh number, $R a$, and the flux ratio, $a$, for given values of the Lewis number, $L e$, and the separation ratio, $\psi$.

\footnotetext{
* Corresponding author: abdeslam_omara@yahoo.fr
} 
A theoretical study of thermo-gravitational separation within a mixture of $\mathrm{N}$ constituents contained in a vertical column is presented by Mutschler et al. [12]. Based on the parallel flow hypothesis, used in the case of binary mixtures, they analytically obtain the velocity, temperature and mass fraction fields within the column. The results concerning theoretical and numerical investigation of species separation in a binary liquid mixture saturating a horizontal porous annulus space where the inner cylinder is heated isothermally was obtained by Abahri et al. [13]. A parametric study based on the Rayleigh number, Lewis number, separation factor, porosity and characteristic size of the annular configuration was performed. In particular, they found that reducing the dimensionless ratio $\left(R=r_{e} / r_{i}\right)$, improve both the maximal separation $\left(S_{\max }\right)$ and $R a_{\mathrm{opt}}$. Bourich et al. [14] considered Soret driven thermosolutal convection in a shallow porous enclosure saturated with a binary fluid. They have found that for large Rayleigh and Lewis numbers, the heat and mass transfer rates exhibit an asymptotic behavior and tend to become independent of these numbers for large Soret coefficient. The separation of the components of a binary mixture in a vertical circular porous annulus has been studied by Bahloul et al. [15]. It was assumed that the vertical walls of the cavity are heated and cooled by constant heat fluxes while the horizontal walls are insulated. Nasrabadi et al. [16] studied numerically species separation in a thermogravitational column filled with a porous medium to predict El Maataoui's experimental data [17]. Their study showed good agreement with El Maataoui's [17] experimental data for both the binary mixture and the ternary mixture. Rtibi et al. [18] studied numerically and analytically the effect of a magnetic field on the species separation in an inclined porous cavity saturated by an electrically conductive binary mixture and provided with four impermeable walls. Their results showed that the magnetic field can enhance the species separation in cases where the optimal coupling between thermosolutal diffusion and convection is not achieved in its absence. However, in cases where this optimal coupling is reached in the absence of the magnetic field, the application of the latter destroys the separation of species. Combined effects of magnetic field and thermodiffusion on natural convection within an electrically conducting binary mixture, in a horizontal porous enclosure subjected to uniform fluxes of heat and mass, is studied analytically and numerically by Bourich et al. [19]. The effect of Hartmann number is depicted graphically and analyzed in detail.

The aim of this work is to study numerically the influence of the inclination angle on the separation of a binary mixture in a cavity subjected to uniform or nonuniform wall temperature under natural convection.

\section{Governing equations}

The system under consideration consists of an inclined rectangular cavity of large aspect ratio $A=L / H$, where $H$ is the height of the cavity along the $y$-axis and $L$ is its length along the $x$-axis (Fig. 1). The cavity is filled with a binary mixture of density $\rho$ and dynamic viscosity $\mu$. The impermeable wall at $y=0$ is kept at cold temperature $\mathrm{T}_{\mathrm{C}}$ while the impermeable wall at $y=\mathrm{H}$ is kept at uniform $\left(\mathrm{T}_{\mathrm{H}}\right)$ or non-uniform $\left(\mathrm{T}_{\mathrm{H}}(x)\right)$ hot temperatures. The walls at $x=0$ and $x=\mathrm{L}$ are impermeable and adiabatic. The binary mixture is assumed to be Newtonian and satisfy the Boussinesq approximation. Thus, the density of the fluid mixture, $\rho$, is a function of the local temperature, $T$, and the local mass fraction, $C$. A linear variation of $\rho$ with $T$ and $C$ is assumed, which is reasonable for small temperature and mass fraction differences (eq. 1):

$$
\rho=\rho_{0}\left[1-\beta_{T}\left(T-T_{0}\right)-\beta_{C}\left(C-C_{0}\right)\right]
$$

The thermal and mass expansion coefficients $\left(\beta_{\mathrm{T}}, \beta_{\mathrm{C}}\right)$ are defined as follows:

$$
\beta_{T}=-\frac{1}{\rho}\left(\frac{\partial \rho}{\partial T}\right), \beta_{C}=-\frac{1}{\rho}\left(\frac{\partial \rho}{\partial C}\right)
$$

Subject to these hypotheses, the governing conservation equations for mass, momentum, energy and chemical species in dimensionless form are:

$$
\begin{gathered}
\frac{\partial U}{\partial X}+\frac{\partial V}{\partial Y}=0 \\
U \frac{\partial U}{\partial X}+V \frac{\partial U}{\partial Y}=-\frac{\partial P}{\partial X}+\operatorname{Pr}\left(\frac{\partial^{2} U}{\partial X^{2}}+\frac{\partial^{2} U}{\partial Y^{2}}\right)+\operatorname{RaPr}[\theta- \\
\psi C] \cos (\alpha) \\
U \frac{\partial V}{\partial X}+V \frac{\partial V}{\partial Y}=-\frac{\partial P}{\partial Y}+\operatorname{Pr}\left(\frac{\partial^{2} V}{\partial X^{2}}+\frac{\partial^{2} V}{\partial Y^{2}}\right)+\operatorname{Ra} \operatorname{Pr}[\theta- \\
\psi C](-\sin (\alpha)) \\
U \frac{\partial \theta}{\partial X}+V \frac{\partial \theta}{\partial Y}=\left(\frac{\partial^{2} \theta}{\partial X^{2}}+\frac{\partial^{2} \theta}{\partial Y^{2}}\right) \\
U \frac{\partial \theta}{\partial X}+V \frac{\partial \theta}{\partial Y}=\frac{1}{L e}\left(\frac{\partial^{2} C}{\partial X^{2}}+\frac{\partial^{2} C}{\partial Y^{2}}\right)+\frac{1}{L e}\left(\frac{\partial^{2} \theta}{\partial X^{2}}+\frac{\partial^{2} \theta}{\partial Y^{2}}\right)
\end{gathered}
$$

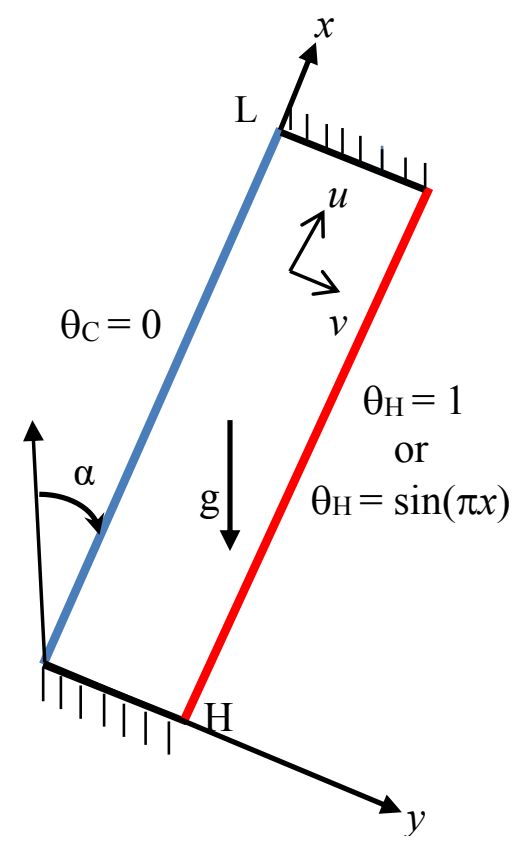

Fig. 1. Geometry and boundary conditions of the problem considered

Noting that in equation (7), $\mathrm{C}$ is the dimensionless concentration of the heaviest component. 
In this study, a positive separation ratio $\psi$ is considered, which implies that, due to thermo-diffusion, the denser and the heavy components of the mixture migrate toward the cold and the hot walls, respectively.

In these equations:

$$
\begin{gathered}
X, Y)=\frac{(x, y)}{H},(U, V)=\frac{(u, v)}{V_{\text {ref }}}, P=\frac{p}{P_{\text {ref }}}, \theta=\frac{T-T_{c}}{\Delta T}, C= \\
\left(C-C_{0}\right) / \Delta C, \Delta T=T_{h}-T_{c}, \\
\Delta C=\Delta T \times C_{0}\left(1-C_{0}\right) \times \frac{D_{T}}{D}
\end{gathered}
$$

where $V_{\text {ref }}=\frac{a}{H}, P_{\text {ref }}=\rho_{0} \frac{a^{2}}{H^{2}}$

The associated non-dimensional boundary conditions over the walls of the enclosure are:

$\left\{\begin{array}{l}U(Y=0 \text { or } 1, X)=U(Y, X=(0, A))=0 \\ V(Y=0 \text { or } 1, X)=V(Y, X=(0, A))=0\end{array}\right.$

$\left\{\right.$ Right hot wall: $\theta(Y=1, X)=\theta_{H}=$

$\left\{\begin{array}{cc}1 & \text { (uniform wall temperature) } \\ \sin \left(\frac{\pi}{A} X\right) & \text { (non uniform wall temperature) }\end{array}\right.$

$\left\{\right.$ Left cold wall: $\theta(Y=0, X)=\theta_{C}=0$

$\frac{\partial C(X, Y)}{\partial X}=\frac{\partial T(X, Y)}{\partial X}=0$ for $X=(0, A) \forall Y \in[0,1]$

$\frac{\partial C(X, Y)}{\partial Y}+\frac{\partial T(X, Y)}{\partial Y}=0$ for $Y=(0,1) \forall X \in[0, A]$

\section{Numerical solution and validation}

The governing partial differential equations (3)-(7), along with the hydrodynamic, thermal and solutal boundary conditions (8)-(12), are solved numerically using the SIMPLE algorithm Patankar [20]. The finite volume method is used to discretize the governing equations. The discretization is done using the powerlaw scheme as given by Patankar [20]. The resulting set of discretized equations for each variable is solved by an iterative process. The iterations are repeated until the convergence criterion:

$\left(\phi_{i, j}^{\mathrm{k}+1}-\phi_{\mathrm{i}, \mathrm{j}}^{\mathrm{k}}\right) / \phi_{\mathrm{i}, \mathrm{j}}^{\mathrm{k}+1} \leq 10^{-5}$ is satisfied for each variables $U, V, C$ and $\theta$ ( $n$ is the iteration number). The spatial resolution is $50 \times 140$, respectively in the $y$ and $x$ directions as one can see it in Fig. 2.

To examine the validity of the numerical simulation, the numerical results relating to the separation obtained in this work are compared with analytical solution and numerical results of Khouzam et al. [1] for two values of mass Rayleigh number, $\mathrm{Ra}_{\mathrm{m}}=537.5$ and $\mathrm{Ra}_{\mathrm{m}}=-400$ for the case with $L e=100, \psi=0.1, \operatorname{Pr}=10$ and $A=10$, Figure 3. As one can see, our numerical results are in good agreement with the analytical and numerical ones of Khouzam et al. [1].

\section{Results}

In this investigation, we try to clarify the impact of uniform and non uniform variation of the temperature of the hot wall on the separation of species in the case of natural convection. The controlling parameters of this investigation are the tilting angle of the cavity, mass Péclet number, $\mathrm{Pe}_{\mathrm{m}}$, and mass Rayleigh numbe, $\mathrm{Ra}_{\mathrm{m}}$.

Figures 4-9 show separation variation, $S=m \times A$ ( $m$ is the concentration gradient between the two ends of the cavity) versus the tilt angle for various values of mass Rayleigh number for the two above mentioned thermal boundary conditions (uniform and non uniform hot wall temperature) at $L e=100, \operatorname{Pr}=10, A=10$ and $\psi=0.1$. It should be noted that if $\alpha=-90^{\circ}$ the cell is heated from above whereas if $\alpha=90^{\circ}$ the cell is heated from below. Figure 4 indicates that the maximum separation is obtained in the case with vertical cell $\left(\alpha=0^{0}\right)$ for mass Rayleigh number equal to 10 regardless of the thermal boundary condition. When the tilting angle approach $90^{0}$ or $+90^{0}$ the separation becomes zero. When mass Rayleigh number, $\mathrm{Ra}_{\mathrm{m}}$, is increased to 50 (Figure 5), the maximum separation spreads over a wide range of tilting angle. Thus, the optimal coupling between thermal diffusion and convection is valid over a wide range of tilting angle in this case contrary to the case with $\mathrm{Ra}_{\mathrm{m}}=$ 10 where we have observed that it is only valid at $\alpha=0^{0}$. By increasing the mass Rayleigh number up to 50, the separation prediction of species undergoes a continuous decrease over a range of the tilting angle values centered around $\alpha=0^{0}$ (vertical position) as can be seen in Figures 6-9.

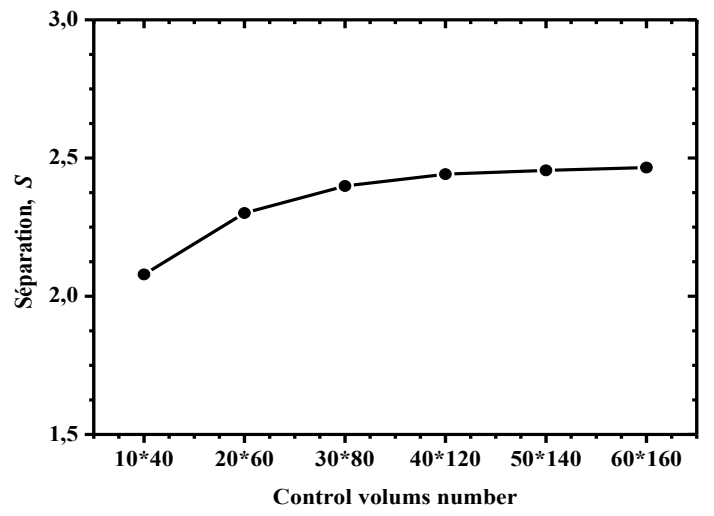

Fig. 2. Separation under different grid number for the case with $\mathrm{A}=10, \mathrm{Ra}=20, \mathrm{Le}=100, \operatorname{Pr}=10, \psi=0.1, \mathrm{Pe}_{\mathrm{m}}=0.03, \alpha=0^{\circ}$.

It is also shown in these figures that as the mass Rayleigh number increase, the maximum of separation is shifted towards high (low) values of the tilting angle with a sharp decrease toward zero at $\alpha=90^{\circ}$ or $-90^{\circ}$. Thus, optimal coupling between thermal diffusion and convection is achieved at these two locations instead of the vertical position for the case with low values of $\mathrm{Ra}_{\mathrm{m}}$. This result has been found by Elhajjar et al. [4] and Platten et al. [5].

It is worth mentioning that the corresponding separation relative to non-uniform heating still lower than the corresponding one of uniform heating regardless of the mass Rayleigh number and the tilting angle. 

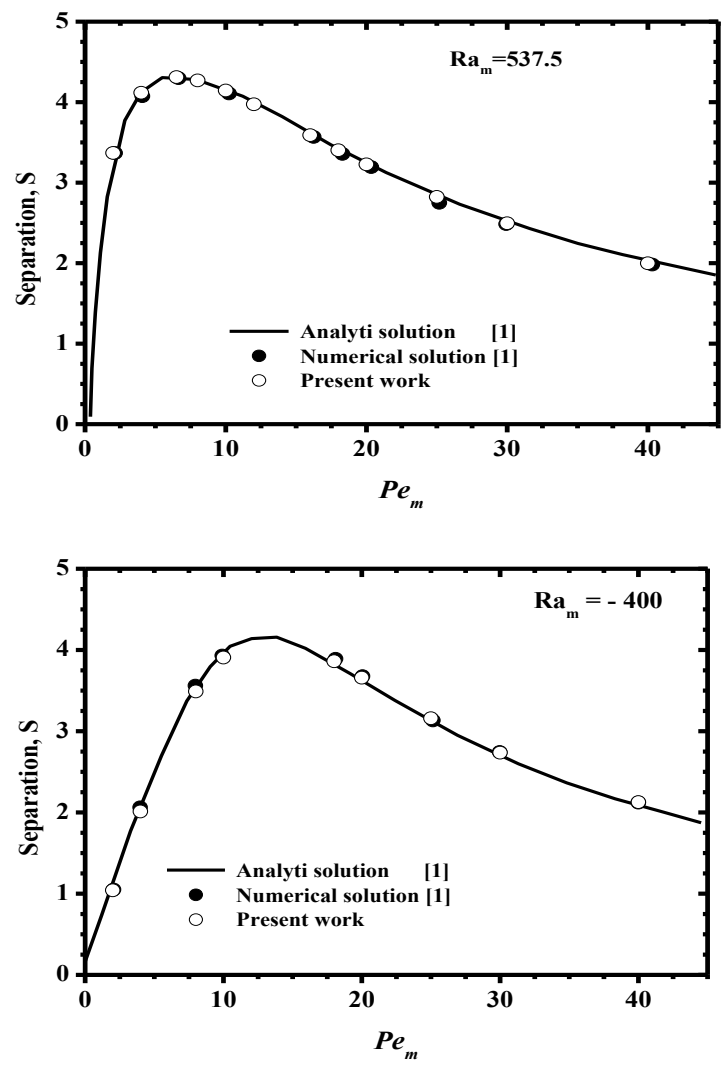

Fig. 3. Comparison of separation obtained numerically in the present work against the analytical and numerical solutions of Khouzam et al. [1] for $L e=100, \operatorname{Pr}=10, A=10, \psi=0.1$.

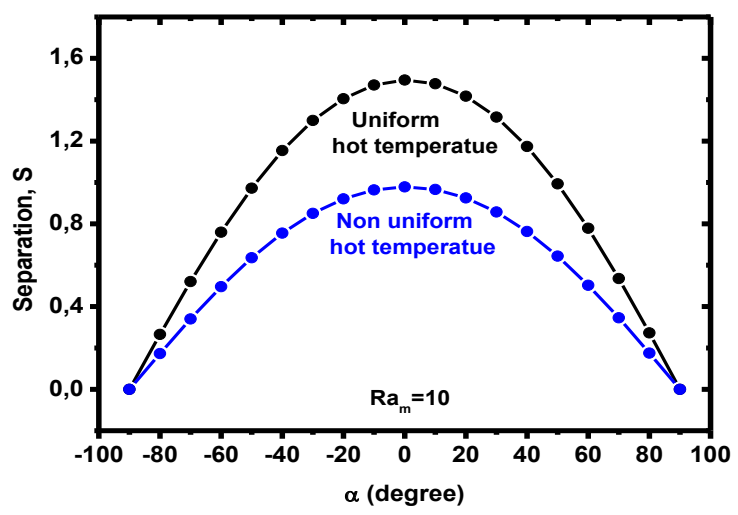

Fig. 4. Variation of the separation with the tilt angle for $\mathrm{Ra}_{\mathrm{m}}=10$.

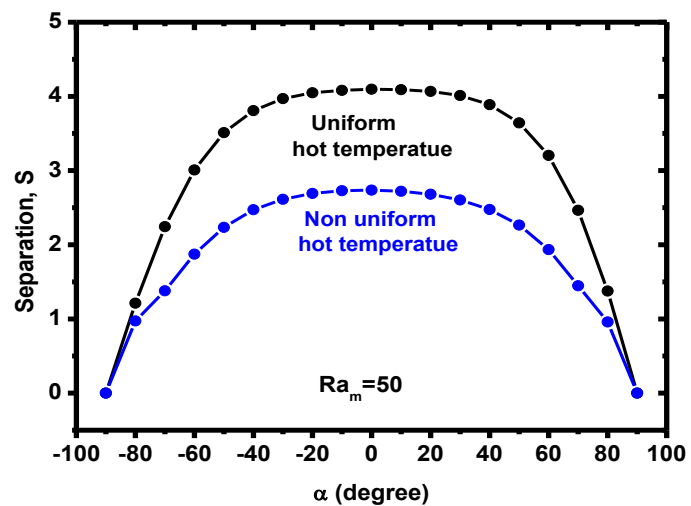

Fig. 5. Variation of the separation with the tilt angle for $\mathrm{Ra}_{\mathrm{m}}=50$.

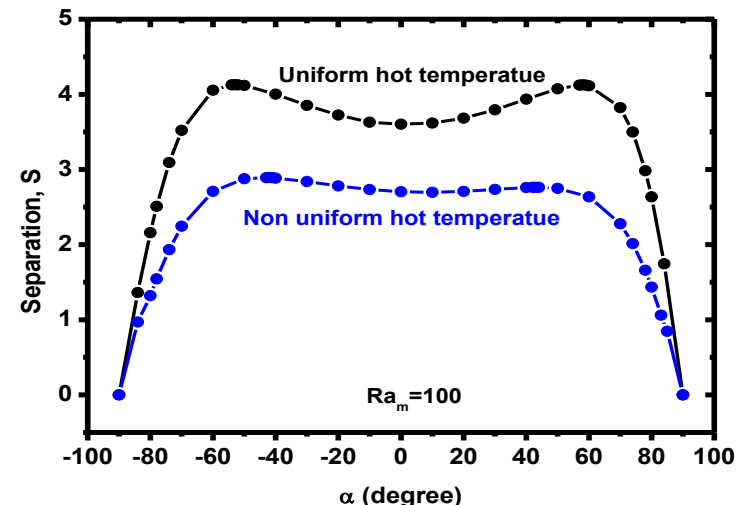

Fig. 6. Variation of the separation with the tilt angle for $\mathrm{Ram}_{\mathrm{m}}=100$

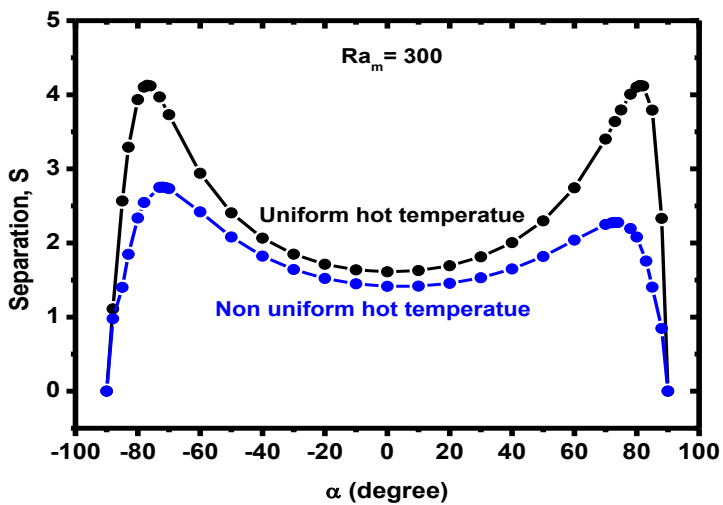

Fig. 7. Variation of the separation with the tilt angle for $\mathrm{Ra}_{\mathrm{m}}=300$

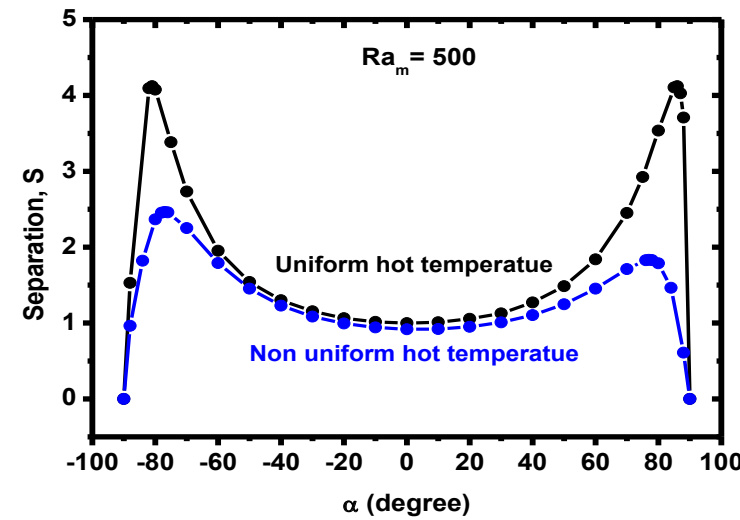

Fig. 8. Variation of the separation with the tilt angle for $\mathrm{Ra}_{\mathrm{m}}=500$.

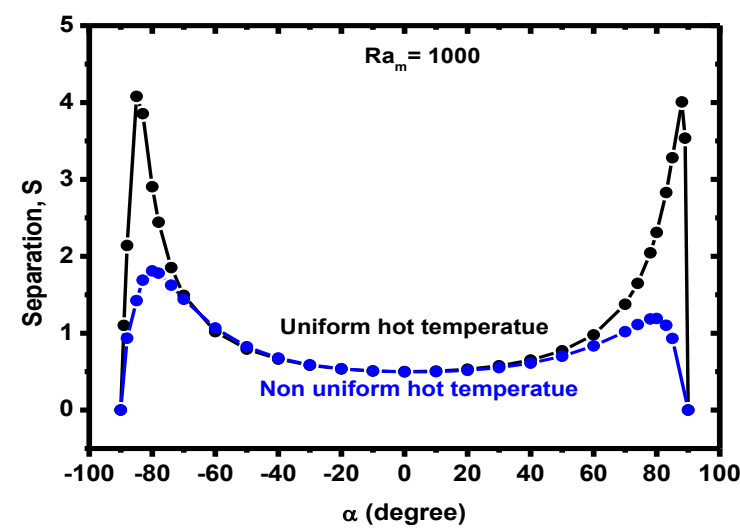

Fig. 9. Variation of the separation with the tilt angle for $\mathrm{Ra}_{\mathrm{m}}=1000$ 
The effect of mass Rayleigh number on the species separation is illustrated in Figure 10 for the case with vertical cavity $\left(\alpha=0^{0}\right)$. For low value of $\mathrm{Ra}_{\mathrm{m}}$, the separation is mainly due to the thermo-diffusion and the role of natural convection is almost small resulting in a small value of species separation. As $\mathrm{Ra}_{\mathrm{m}}$ increases, natural convection velocity raises leading to an increase of mass transfer rate. Therefore, we approach increasingly the optimal coupling between thermodiffusion and convection and $\mathrm{S}$ reach a maximum value. By increasing $\mathrm{Ra}_{\mathrm{m}}$ the separation of species undergoes a continuous decrease until it reaches complete mixing, where no separation takes place. Besides, the separation becomes the same regardless of the type of thermal boundary conditions (uniform or non uniform hot wall temperature).

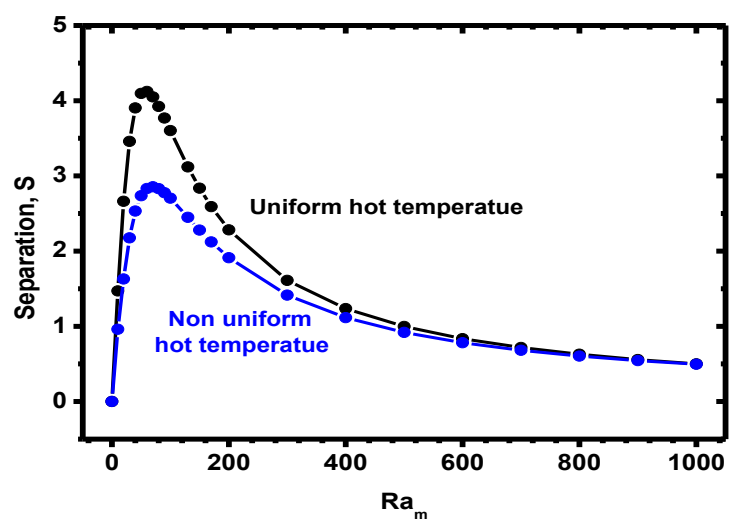

Fig. 10. Effect of mass Rayleigh number on separation in a vertical cavity $\left(\alpha=0^{0}\right)$ for the two type of heating.

Furthermore, the effect of the Lewis number, varied in the range from 10 to 200 , on the optimum Rayleigh number leading to the maximum separation of specifies is shown in Figure 11. Recall that the number of Lewis provides a measure of thermal diffusivity of a fluid to its mass diffusivity. Results shown in this figure indicate that as Lewis values increase, the optimum Rayleigh number undergoes sharp decreases towards zero. Besides, the optimum Rayleigh number, $\mathrm{Ra}_{\mathrm{opt}}$, is almost the same whatever the hot wall temperature is uniform or non uniform except at $L e=10$.

In order to consider the effect of Lewis number on the species separation in the case where the cavity is vertical $\left(\alpha=0^{\circ}\right)$, Figures 12 and 13 show the evolution of separation as a function of thermal Rayleigh number. It can be observed in these figures that the maximum separation value is practically independent on the Lewis number. Moreover, the optimum Ra number is inversely proportional to the Lewis number. Consequently, the optimal Rayleigh number decrease as the Lewis number increases. These behaviors are valid regardless of the boundary conditions (uniform or non uniform hot wall temperature).

To highlight the effects caused by changing the tilting angle and the Lewis number on the separation, Figures 14-15 depicts the separation as a function of $R a$ following three values of $L e$ number for the case with $\left(\alpha=-86^{0}\right)$.
We recall, as we have seen previously, that when the number of $\mathrm{Ra}_{\mathrm{m}}$ increases, the maximum value of the separation is shifted towards high (low) values of the tilting angle which justifies the choice of this angle. Though the preceding observations, relating to Figures 12 and 13 , remain valid, one can observe in this case that the optimal Rayleigh number is increased.

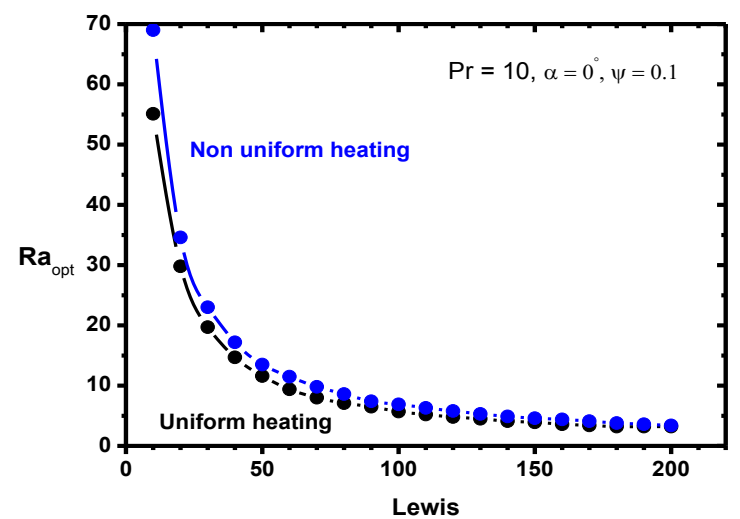

Fig. 11. Effect of Lewis number on the optimal Rayleigh number in a vertical cavity $\left(\alpha=0^{0}\right)$ for the two type of heating

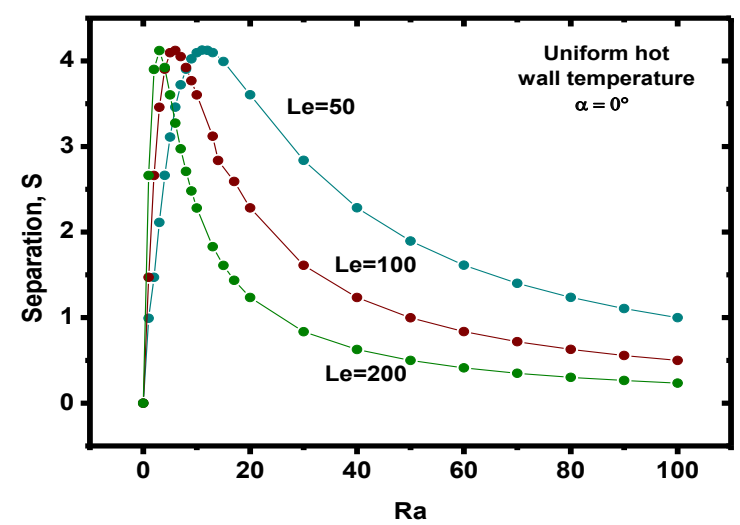

Fig. 12. Effect of Lewis number on separation curves in a vertical cavity $\left(\alpha=0^{0}\right)$ in case with uniform hot wall temperature.

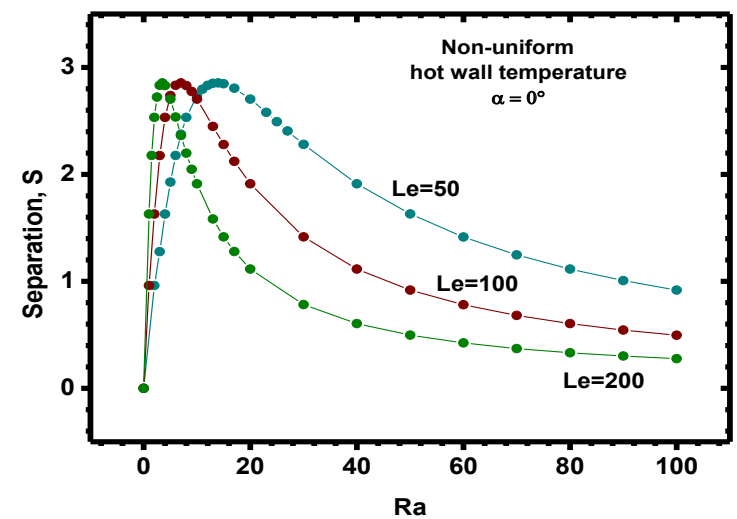

Fig. 13. Effect of Lewis number on separation curves in a vertical cavity $\left(\alpha=0^{0}\right)$ in case with non uniform hot wall temperature.

The results relative to the case with positive tilting angle $\left(\alpha=86^{\circ}\right)$ and uniform hot wall temperature show a decreases of the optimal Rayleigh number for $L e=50$ and 100 compared to the case with $\left(\alpha=-86^{\circ}\right)$ whereas 
for $L e=200$, the optimal Rayleigh number is almost the same, as can be seen in Figure 16.

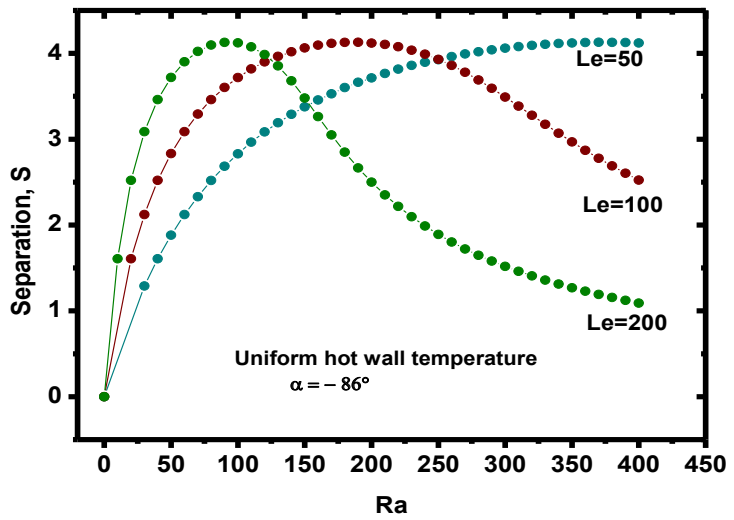

Fig. 14. Effect of Lewis number on separation curves in an inclined cavity $\left(\alpha=-86^{\circ}\right)$ in case with uniform hot wall temperature.

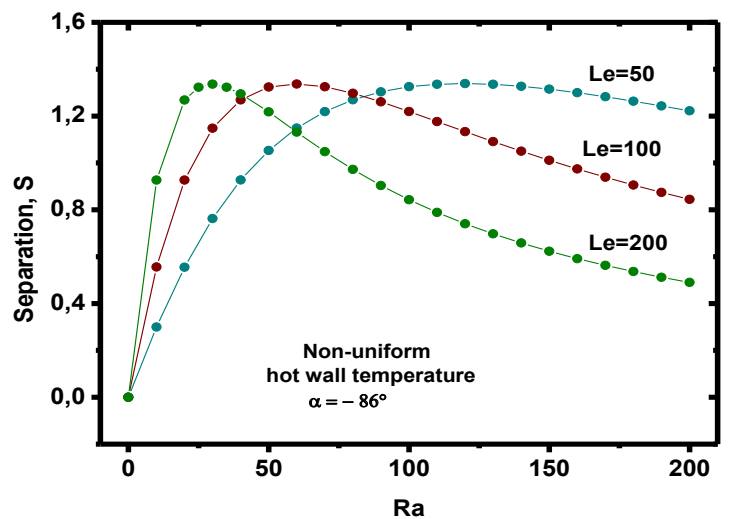

Fig. 15. Effect of Lewis number on separation curves in an inclined cavity $\left(\alpha=-86^{0}\right)$ in case with non uniform hot wall temperature

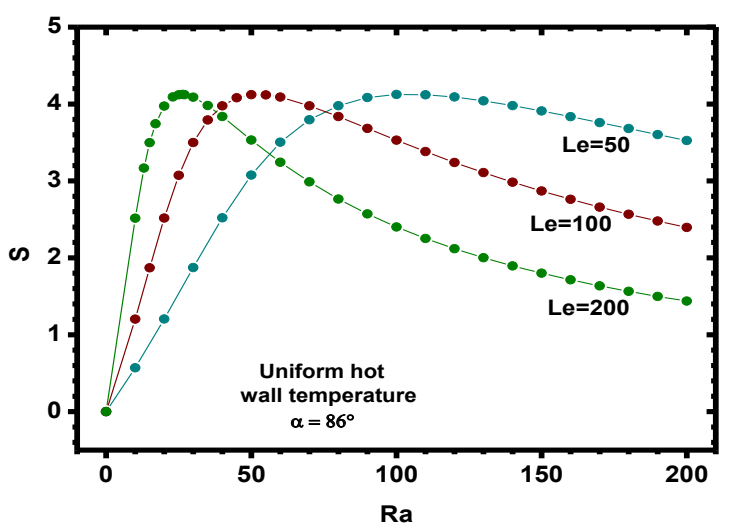

Fig. 16. Effect of Lewis number on separation curves in an inclined cavity $\left(\alpha=86^{0}\right)$ in case with uniform hot wall temperature.

The effect of aspect ratio on the optimum Rayleigh number for the aforementioned modes of heating is given in Table 1. A decrease of the optimum Rayleigh number with the increase of aspect ratio is observed. However, the sensitivity of the optimum Rayleigh number to the aspect is more pronounced in the case with non-uniform heating than the case with uniform heating.
Table1. Effect of the aspect ratio $A$ on the optimal mass Rayleigh number for $\mathrm{Le}=100, \operatorname{Pr}=10, \psi=0.1, \alpha=0^{0}$.

\begin{tabular}{|l|cc|cc|}
\cline { 2 - 5 } \multicolumn{1}{c|}{} & \multicolumn{3}{c|}{ Uniform heating } & \multicolumn{2}{c|}{ Non-uniform heating } \\
\cline { 2 - 5 } \multicolumn{1}{c|}{} & Ram $_{\text {opt }}$ & Separation & Ram $_{\text {opt }}$ & Separation \\
\hline $\mathbf{A = 5}$ & $\mathbf{6 1}$ & $\mathbf{2 . 0 8 9 6}$ & $\mathbf{7 2}$ & $\mathbf{1 . 5 8 7 5}$ \\
\hline $\mathbf{A = 1 0}$ & $\mathbf{5 7}$ & $\mathbf{4 . 1 2 7 4}$ & $\mathbf{6 9}$ & $\mathbf{2 . 8 5 5 4}$ \\
\hline $\mathbf{A = \mathbf { 2 0 }}$ & $\mathbf{5 5}$ & $\mathbf{7 . 9 9 5 2}$ & $\mathbf{6 6}$ & $\mathbf{5 . 4 1 0 0}$ \\
\hline
\end{tabular}

\section{Conclusion}

In this work, numerical studies have been performed to investigate the effect of mass Rayleigh number, tilting angle, Lewis number and aspect ratio on the species separation in a binary fluid mixture inside a rectangular cell. Two different heating modes were considered in this study (uniform and non uniform hot wall temperature). The results show that: 1) At low $\mathrm{Ra}_{\mathrm{m}}$, the separation relative to non-uniform heating still lower than the corresponding one of uniform heating regardless of Lewis number and tilting angle; 2) $\mathrm{As}^{\mathrm{Ra}} \mathrm{m}$ increases, the separation becomes the same for the two modes of heating (uniform and non uniform heating) on a wide range of values of the tilting angle $\alpha$ that extend from $\alpha=-70^{\circ}$ to $\alpha=50^{\circ}$ at $\mathrm{Ra}_{\mathrm{m}}=1000$ while the maximum, observed at $\alpha=0^{0}$ for low $\mathrm{Ra}_{\mathrm{m}}$, is shifted towards high (low) values of the tilting angle with a sharp decrease towards zero at $\alpha=90^{\circ}$ or $-90^{\circ}$;3) Furthermore, the mass Rayleigh number is seen to be weakly affected when the aspect ratio, A, vary from 5 to 20 for the two heating modes.

\section{References}

1. A. Khouzam, A. Mojtabi, M. C. Charrier-Mojtabi, B. Ouattara, Int. J. of Thermal Sciences 73, 18 (2013)

2. R. Bennacer, A. Mahidjiba, P. Vasseur, H. Beji, R. Duval, Int. J. Numer. Methods Heat Fluid Flow 13, 199 (2003)

3. B. Elhajjar, A. Mojtabi, M. Marcoux, M. C. CharrierMojtabi, C. R. Mecanique, 334, 621 (2006)

4. B. Elhajjar, A. Mojtabi, P. Costesque, M. C. Charrier-Mojtabi, Int. J. Heat and Mass Transfer, 53, 4844 (2010)

5. J. K. Platten, M. M. Bou-Ali, J. F. Dutrieux, J. Phys. Chem. B, 107, 11763 (2003)

6. S. Ouadhani, A. Abdennadher, A. Mojtabi, Eur. Phys. J. E, 40, 38 (2017)

7. M. Yang, M. Ripoll, J. Phys.: Condens. Matter, 24, 195 (2012)

8. V. M. Shevtsova, D. E. Melnikov, J. C. Legros, Physical Review E, 73, 047302 (2006)

9. D. Henry, B. Roux, J. Fluid Mech. 195, 175 (1988)

10. M. C. Raju, S. V. K. Varma, P. V. Reddy, S. Saha, J. of Mechanical Engineering, ME39 (2), 65 (2008)

11. L. Yacine, A. Mojtabi, R. Bennacer, A. Khouzam, Int. J. of Thermal Sciences, 104, 29 (2016) 
12. D. Mutschler, M. A. Larabi, A. Mojtabi, 23 ème Congrès Français de Mécanique, Lille (France), 28 août -1 septembre (2017)

13. O. Abahri, D. Sadaoui, K. Mansouri, A. Mojtabi, M. C. Mojtabi, Mechanics \& Industry 18, 106 (2017)

14. M. Bourich, M. Hasnaoui, A. Amhamid, M. Mamou, Int. Comm. Heat Mass Transfer, 29(5), 717 (2002)

15. A. Bahloul, M. A. Yahiaoui, P. Vasseur, R. Robillard, Int. Comm. Heat Mass Transfer, 31(6), 783 (2004)

16. H. Nasrabadi, H. Hoteit, A. Firoozabadi, Transp Porous Med, 67, 473 (2007)

17. M. El Maataoui, PhD dissertation, U. Paul Sabatier, Toulouse, France (1986).

18. A. Rtibi, M. Hasnaoui, A. Amahmid, J. of Applied Fluid Mechanics, 11(4), 1059 (2018)

19. M. Bourich, M. Hasnaoui, A. Amahmid, M. ErRaki, A. Lagra1, M. Mamou, J. of Applied Fluid Mechanics, 9(2), 741 (2016

20. S. V. Patankar, Numerical Heat Transfer and Flow, Hemisphere Publication Company, New York (1980) 